\title{
Trois modèles de revenu de base
}

\section{Pierre-Étienne Vandamme, Raisons politiques, 83, 2021, p. 17-29.}

\section{Introduction}

Le revenu de base (revenu universel, d'existence, de citoyenneté, ou encore allocation universelle) fait partie de ces sujets sur lesquels tant les académiques que les acteurs politiques se sentent tenus de prendre position, si possible de manière ferme. Pourtant, être pour ou contre le revenu de base n'a pas grand sens. Il s'agit en effet d'un instrument, comme la fiscalité, qui peut être mis au service d'une grande diversité de visées politiques, ce qui explique qu'il soit défendu autant par des libertariens de droite que par des libéraux plus modérés, des socialistes, des républicains ou encore des écologistes ${ }^{1}$. Si l'on souhaite évaluer un tel instrument d'action publique d'un point de vue normatif, il faut donc nécessairement sortir de l'abstraction pour examiner une proposition concrète et le projet politique qui l'accompagne (qu'il soit explicite ou non).

Dans cet esprit, le présent article vise à distinguer et comparer trois idéaux-types de revenu de base : «néolibéral », « social-démocrate » et « de transition écologique et sociale », auxquels on pourrait associer respectivement, à titre illustratif, les figures de Milton Friedman, Thomas Piketty et Philippe Van Parijs ${ }^{2}$. Ces idéaux-types se distinguent essentiellement par leurs caractéristiques internes (montant; financement; articulation avec les aides sociales existantes) et par les projets politiques qui les guident. Ils sont présentés tour à tour dans les sections 2 à 4 puis comparés dans la section 5. Cela permettra d'examiner leurs radicalités respective et commune. Nous verrons également qu'il existe une fracture irréconciliable entre le modèle «néolibéral » et les deux autres qui, en revanche, ne sont pas nécessairement incompatibles.

L'objectif principal de cet article est de « politiser » les débats sur le revenu de base. Il arrive fréquemment que ses partisans le présentent, pour séduire un maximum de lecteurs, comme une proposition transcendant le clivage gauche-droite ${ }^{3}$, voire susceptible d'un

\footnotetext{
${ }^{1}$ Voir Philippe Van Parijs (dir.) Arguing for Basic Income: Ethical Foundations for a Radical Reform, Londres, Verso, 1992 ; Karl Widerquist, José A. Noguera, Yannick Vanderborght \& Jurgen De Wispelaere (dir.), Basic Income: An Anthology of Contemporary Research, Chichester, Wiley-Blackwell, 2013.

${ }^{2}$ Avec deux nuances, toutefois, puisque Piketty serait favorable à une transition vers un au-delà du capitalisme (une forme nouvelle de propriété sociale), tandis que Van Parijs a également défendu la version «socialedémocrate » du revenu de base, comme nous le verrons plus loin.

${ }^{3}$ Rutger Bergman, Utopies réalistes, Paris, Seuil, 2017.
} 
consensus par recoupement entre courants philosophiques très variés. Si une telle approche a sans doute des attraits rhétoriques, elle masque la diversité des modèles et projets qui peuvent sous-tendre une telle proposition, rendant l'évaluation plus difficile. De même, il est fréquent que les adversaires du revenu de base le dépeignent comme une proposition unique, qui posséderait nécessairement les traits qui leur déplaisent. Que l'on songe par exemple à la critique de gauche pointant systématiquement du doigt la filiation avec Milton Friedman ${ }^{4}$, qui ne défendait pourtant qu'une version très particulière et très conservatrice du revenu de base (un très maigre impôt négatif, plus précisément). De telles approches de la question obscurcissent les débats. Il me semble que la manière la plus intéressante d'aborder la question du revenu de base est d'en reconnaître la diversité des formes et de les évaluer séparément. Politiser le débat, dans cette perspective, ne signifie pas adopter une lecture partisane de celuici ou renoncer à la nuance philosophique, mais dévoiler la diversité des projets politiques sousjacents au débat et les tensions entre ceux-ci. Et c'est à une telle démarche que cet article entend contribuer ${ }^{5}$.

Avant d'entrer dans le vif du sujet, trois précisions me semblent importantes. Premièrement, j'entends par « revenu de base » un revenu octroyé par l'État à titre individuel (sans égard pour la composition de ménage) et de façon inconditionnelle (dans le sens où aucune contrepartie n'est exigée), mais pas forcément universelle ${ }^{6}$. Soit un revenu est distribué à l'ensemble des citoyens, mais absorbé progressivement par l'impôt, en fonction des revenus -

\footnotetext{
${ }^{4}$ Mateo Alaluf, L'Allocation universelle. Nouveau label de précarité, Bruxelles, Couleur Livres, 2014 , p. 14 et 80 ; Daniel Zamora, «Histoire et genèse d'une idée néolibérale », dans M. Alaluf et D. Zamora (dir.), Contre l'allocation universelle, Québec, Lux, 2016.

${ }^{5}$ Il n'est pas le premier à proposer une typologie de ce type (centrée sur les modèles politiques plutôt que les approches philosophiques ou théories de la justice). Voir notamment Guillaume Allègre, « Revenus universels », Revue de l'OFCE, 154, 2017, p. 7-9 ; Marc-Antoine Sabaté, « Le revenu de base : renversement ou renouveau du droit social ? Éléments pour une philosophie politique et sociale de l'inconditionnalité », thèse de doctorat, Université libre de Bruxelles, 2020, p. 61-66. À ma connaissance, toutefois, la confrontation de ces différents modèles n'a pas encore été approfondie. Dans les travaux mentionnés ci-dessus, ces distinctions ne sont évoquées que brièvement.

${ }^{6}$ C'est une définition qui n'est pas consensuelle (en raison notamment du succès des appellations « revenu universel » et « allocation universelle »), mais de plus en plus fréquente. Voir par exemple Julia Cagé, « Le Revenu universel comme réforme réaliste de la protection sociale. Entretien avec Julia Cagé », dans G. Allègre \& P. Van Parijs (dir.), Pour ou contre le revenu universel ?, Paris, PUF, 2018, p. 23-32; Thomas Piketty, Capital et idéologie, Paris, Seuil, 2019 ; Marc-Antoine Sabaté, « Revenu de base inconditionnel : quel instrument pour quelle justice fiscale ? Réflexions à partir du cas des « gilets jaunes » en France », Éthique publique 21 (2), 2019.
} 
formule «allocation universelle »-, soit le revenu octroyé décroît (jusqu'à zéro) en fonction des revenus et n'est donc distribué qu'aux chômeurs et bas revenus - formule « impôt négatif » ou «crédit d'impôt remboursable ». Dans les deux cas, l'idée essentielle est de garantir un certain seuil de revenu à chaque citoyen tout en facilitant le travail rémunéré. Qu'il soit pour cela préférable de passer par une distribution universelle ou plus ciblée est une question secondaire ${ }^{7}$, que je souhaite laisser à l'arrière-plan. Cela permet d'envisager et de comparer un plus grand nombre de propositions. Nous verrons en effet que le modèle «néolibéral» s'accommode assez mal de l'universalité, et que ce n'est pas non plus l'option la plus plausible du point de vue « social-démocrate ».

Deuxièmement, la typologie proposée ici ne prétend pas être exhaustive. Il existe à n'en pas douter des modèles de revenu de base qui échappent à ces trois idéaux-types. Je me concentre ici sur les modèles les plus souvent défendus parmi ceux qui s'intègrent dans l'appareil redistributif de l'État social. Je laisse donc notamment de côté le modèle dont la filiation remonte à Thomas Paine et qui consiste à redistribuer de manière égale à toutes et tous les bénéfices de l'exploitation de ressources naturelles, comme c'est le cas depuis plusieurs années en Alaska ${ }^{8}$. Je me concentre plutôt sur les propositions de réforme de l'État social.

Troisièmement, comme toute typologie, celle-ci est imparfaite : certaines propositions de revenu de base pourront être difficiles à placer entre deux idéaux-types. C'est d'autant plus possible qu'un certain nombre de défenseurs du revenu de base rechignent à politiser clairement leur proposition, pour séduire un maximum de lecteurs. Davantage encore qu'une description du champ des propositions existantes, la typologie offerte ici est donc une invitation aux défenseurs du revenu de base à clarifier quel modèle ils promeuvent - et aux opposants à clarifier quel modèle ils attaquent. Malgré son caractère inévitablement imparfait, la typologie proposée me semble de nature à éclaircir les débats, les trois idéaux-types étant suffisamment distincts et cohérents pour permettre l'analyse de trois projets politiques bien différents.

\section{Le revenu de base « néolibéral »}

\footnotetext{
${ }^{7}$ Cela ne signifie pas qu'elle ne soit pas importante, mais je souhaite simplement déplacer l'attention vers l'essentiel de la proposition, à savoir la garantie inconditionnelle d'une sécurité de revenu.

${ }^{8}$ Voir Karl Widerquist \& Michael Howard (dir.), Alaska's permanent fund dividend: examining its suitability as a model, New York, Palgrave Macmillan, 2012.
} 
Le terme «néolibéral » est plus souvent invoqué que défini, et utilisé pour désigner des réalités très diverses, généralement de manière péjorative. Je l'utilise ici pour désigner un projet politique combinant la réduction des interventions et dépenses publiques et la flexibilisation du marché de l'emploi en vue de promouvoir la liberté de marché et, à travers elle, la croissance économique. Promouvoir la croissance n'est pas propre au néolibéralisme. Réduire les dépenses publiques et flexibiliser le marché de l'emploi non plus. Mais combiner ces deux moyens au service de cette fin, de manière prioritaire par rapport à d'autres visées comme la préservation de conditions de vie décentes pour les plus démunis ou de l'égalité des chances est caractéristique de l'approche que je qualifierai ici de néolibérale ${ }^{9}$, de même que la forte réticence à l'élargissement de la sphère d'intervention de l'État au-delà de la protection de la liberté de marché. Le présupposé central du néolibéralisme est que «le marché est bien plus capable que l'État de résoudre les problèmes et de satisfaire les besoins des êtres humains ${ }^{10} »$. Pour cette raison, les politiques sociales (s'il y en a), doivent interférer aussi peu que possible avec la liberté de marché ${ }^{11}$.

On peut associer à ce premier modèle la figure de Milton Friedman ${ }^{12}$, mais aussi plus récemment Charles Murray ${ }^{13}$ ou Matt Zwolinski ${ }^{14}$. Dans une telle perspective, ce qui séduit dans l'idée d'un revenu de base garanti pour chacun de manière inconditionnelle, c'est d'abord la simplification du régime redistributif de l'État social : la fusion de la plupart des dépenses

\footnotetext{
${ }^{9}$ Le terme me paraît préférable à « libéral », souvent utilisé en France pour désigner le libéralisme économique, mais qui est parfois utilisé dans un sens fort différent (comme dans l'égalitarisme libéral d'un John Rawls ou d'un Philippe Van Parijs, par exemple), en particulier dans les débats sur le revenu de base. Et il me paraît préférable à « libertarien », parce que ce n'est pas tant la liberté individuelle ou la protection de droits de propriété « naturels » qui priment dans l'approche ici décrite que la liberté de marché au service de la croissance, donc à des fins de maximisation des richesses collectives - une perspective qui se rapproche davantage de l'utilitarisme (dans une interprétation « de droite ») que du libertarisme.

${ }^{10}$ Colin Crouch, L'étrange survie du néolibéralisme, Bienne-Berlin, Diaphanes, 2016, p. 20.

${ }^{11}$ Je me concentre ici sur ce seul aspect du néolibéralisme, laissant de côté un autre aspect souvent évoqué dans ses définitions, à savoir la colonisation de toutes les sphères de l'existence par la logique du marché. Voir par exemple Pierre Dardot \& Christian Laval, La nouvelle raison du monde. Essai sur la société néolibérale, Paris, La découverte, 2020.

${ }^{12}$ Milton Friedman, Capitalism and Freedom, Chicago, University of Chicago Press, 2002 (1962), p. 190-195.

${ }^{13}$ Charles Murray, In Our Hands: A Plan to Replace the Welfare State, Washington, AEI Press, 2006.

${ }^{14}$ Matt Zwolinski, « A moral case for Universal Basic Income: A neoliberal argument for UBI based on individual freedom and property rights », The Critic, juillet-août 2020.
} 
sociales en un seul revenu, moins coûteux à administrer ${ }^{15}$. On est donc bien dans la logique de réduction des dépenses publiques - en particulier des dépenses liées à l'accompagnement et à l'activation des personnes sans emploi - et plus globalement d'amincissement de l'État social et de la sphère d'intervention de l'État.

C'est ensuite la flexibilisation du marché de l'emploi que permet la garantie de revenu. En effet, la garantie d'un revenu de remplacement en cas de perte d'emploi peut être vue comme facilitant les licenciements, les reconversions, le travail à temps partiel - des changements temporaires de statut qui peuvent autant être souhaités par les travailleurs que rendus nécessaires par la conjoncture économique. L'essentiel, pour que la fluidité soit réelle dans le passage d'un statut à l'autre est d'éviter lesdits « pièges à l'emploi » auxquels font face les personnes qui, n'ayant accès qu'à des emplois peu rémunérés et devant la perspective de perdre une série d'avantages sociaux et de faire face à de nouvelles dépenses (de garde d'enfants, de transport), sont peu incitées économiquement à reprendre un emploi ${ }^{16}$. Or, c'est ce que permet le revenu de base dès lors que les personnes à faible revenu peuvent le combiner avec les revenus du travail. Chaque heure de travail est donc payante ${ }^{17}-\mathrm{y}$ compris le travail à temps partiel ou ce qu'on appelle désormais les « mini-jobs », au bénéfice du dynamisme économique et de la création de richesses.

Néanmoins, si l'objectif général est de maximiser la création de richesses tout en minimisant les dépenses publiques, il est peu probable qu'une telle approche recommande un revenu de base généreux - qui, par exemple, permettrait de couvrir tous les besoins essentiels des personnes. En effet, même si chaque heure de travail paye, une personne hésitera davantage à accepter un emploi peu gratifiant si elle a de quoi satisfaire ses besoins que si elle est dans la nécessité. Les partisans d'un revenu de base «néolibéral » seront donc probablement en faveur d'un montant relativement faible, préservant une forte incitation à l'emploi - au contraire des deux autres modèles présentés dans ce qui suit.

\footnotetext{
${ }^{15}$ On retrouve également cette motivation de simplification chez des auteurs comme Gaspard Koenig et Marc de Basquiat (LIBER, un revenu de liberté pour tous. Une proposition d'impôt négatif en France, Génération libre, 2014), mais dans une perspective plus modérée que chez les auteurs susnommés.

${ }^{16}$ Cela ne signifie pas que ces personnes refuseront nécessairement de travailler, car la motivation économique n'est pas la seule possible, ni celle qui domine (François Dubet \& Antoine Vérétout, « Une réduction de la rationalité de l'acteur. Pourquoi sortir du RMI ? », Revue française de sociologie, 42 (3), 2001, p. 407-436), mais cela signifie que le retour à l'emploi pourrait être mieux encouragé ou facilité.

${ }^{17}$ Milton Friedman, Capitalism and Freedom, op. cit., p. 192 ; There is no such thing as a free lunch, LaSalle, Open Court, 1975, p. 201.
} 
Quant à l'universalité, elle n'est pas nécessaire dans un tel modèle. Dans une visée de réduction du rôle de l'État et de minimisation des taxes et transferts, elle paraît même absurde si une garantie de revenu plus ciblée peut produire des résultats plus ou moins équivalents ${ }^{18}$.

Enfin, ce modèle se distingue par le fait que le revenu de base n'est pas envisagé comme une mesure redistributive. Il s'agit certes d'une redistribution par rapport à un scénario hypothétique sans aucune aide sociale. Mais par rapport aux systèmes de protection sociale existants, la réforme envisagée n'est pas censée accroître les redistributions. On n'insiste pas, par exemple, sur la progressivité de l'impôt ${ }^{19}$. Il s'agit plutôt de réorganiser le système de transferts existant - pour le simplifier et l'amincir. Et si le modèle vise éventuellement à combattre la pauvreté, il ne s'intéresse pas et ne s'attaque donc pas à la question des inégalités ${ }^{20}$.

\section{Le revenu de base social-démocrate}

J'entends par « social-démocratie » un ensemble de positions politiques d'inspiration socialiste ayant pour ambition de réduire, par la participation au gouvernement, les injustices sociales générées par le capitalisme ${ }^{21}$. Que l'ambition ultime soit encore la socialisation des moyens de production, une autre alternative socialiste ou un idéal révisé de capitalisme « domestiqué », on peut considérer que la social-démocratie a pour visée principale, dans le monde tel qu'il est, la « démarchandisation » de l'accès à la subsistance ${ }^{22}$ et la limitation des effets des inégalités de revenu. Pour ce faire, l'outil principal est l'État social, dont les sociaux-démocrates cherchent surtout, pour le moment, à préserver les acquis. La mission principale de l'État social est de

\footnotetext{
${ }^{18}$ Ce peut être plus ou moins le cas si une avance sur l'impôt négatif est payée mensuellement, sur base des revenus personnels (et pas du ménage). Néanmoins, la moindre lisibilité de l'impôt négatif pourrait engendrer des taux de recours plus faibles (et donc plus de pauvreté), tandis que l'incertitude quant aux compensations auxquelles on aura droit, calculées annuellement, pourrait avoir un effet désincitatif sur le travail. Voir Thomas Piketty, «Allocation compensatrice de revenu ou revenu universel », dans R. Godino et al., Pour une réforme du RMI, Notes de la Fondation Saint Simon, 104, 1999, p. 21-29 ; Philippe Van Parijs \& Yannick Vanderborght, Le revenu de base inconditionnel. Une proposition radicale, Paris, La Découverte, 2019, p. 63-72.

${ }^{19}$ Marc-Antoine Sabaté, «Le revenu de base : renversement ou renouveau du droit social ? », op. cit., p. 65.

${ }^{20} \mathrm{Ce}$ qu'avouent d'emblée, par exemple, Gaspard Koenig et Marc de Basquiat en introduisant leur proposition (LIBER, un revenu de liberté pour tous, op. cit., p. 2-3) et fait l'objet de certaines objections de gauche au revenu de base (par exemple, Daniel Zamora, « The Case Against a Basic Income », Jacobin, 28 décembre 2017).

${ }^{21}$ Adam Przeworski, Capitalism and Social Democracy, Cambridge, Cambridge University Press, 1985.

${ }^{22}$ Gøsta Esping-Andersen, Les trois mondes de l'État-providence. Essai sur le capitalisme moderne, Paris, PUF, 1999 (1990).
} 


\section{Version d'auteur}

protéger les personnes les plus vulnérables ${ }^{23}$ : personnes sans emploi, infirmes, malades, retraitées, ou ayant de nombreux enfants à charge. Il s'agit à la fois de leur garantir une sécurité matérielle en cas d'absence de revenus du travail (suffisants), afin d'éviter la pauvreté, et également de leur offrir une protection contre des formes d'emploi considérées comme inacceptables.

En raison de ces objectifs, l'idée d'un revenu de base généreux distribué à l'ensemble des citoyens ou résidents permanents d'un pays possède un attrait certain. D'abord, parce que fixé à un montant suffisamment élevé et combiné avec des versements particuliers destinés aux catégories les plus vulnérables, ou aux besoins spécifiques, le revenu de base constituerait un instrument très efficace de lutte contre la pauvreté. Du fait de son automaticité, il ferait en effet très probablement chuter le taux de non-recours ${ }^{24}$ : plus grand monde ne passerait entre les mailles du filet de protection sociale. Ensuite, il permettrait de lutter contre le chômage, en incitant au partage du travail et en réduisant les désincitations à l'emploi évoquées plus haut. Par ailleurs, du point de vue des sociaux-démocrates, il offrirait des conditions de vie plus justes à l'ensemble des personnes qui se rendent utiles à la collectivité à travers des activités faiblement ou pas du tout rémunérées ${ }^{25}$.

On peut associer à ce modèle des figures comme Thomas Piketty ${ }^{26}$, Guy Standing ${ }^{27}$ ou Yannick Vanderborght ${ }^{28}$. Dans cette perspective, le revenu de base peut être vu comme un

\footnotetext{
${ }^{23}$ Robert Goodin, Reasons for welfare: The political theory of the welfare state, Princeton, Princeton University Press, 1988.

${ }^{24}$ Philippe Warin, «Qu'est-ce que le non-recours aux droits sociaux ? », La Vie des idées, $1^{\mathrm{er}}$ juin 2010. A fortiori, donc, si le revenu est universel plutôt que ciblé.

${ }^{25}$ À noter que cet objectif peut entrer en tension avec une perspective d'émancipation des femmes par rapport aux tâches domestiques, qu'une approche sociale-démocrate devrait sans doute intégrer. Voir l'article éclairant d'Ingrid Robeyns, « Will a Basic Income Do Justice to Women? », Analyse \& Kritik, 23, 2001, p. 88-105.

${ }^{26}$ Thomas Piketty, Capital et idéologie, op. cit. Précisons que les propositions de Piketty par rapport à la propriété privée vont bien au-delà de propositions sociales-démocrates plus modestes. Comme mentionné plus haut, il est favorable à une transition vers un au-delà du capitalisme. Néanmoins, ce n'est pas le revenu de base, chez lui, qui amorce cette transition. Il n'y voit qu'un instrument modeste de revalorisation des bas revenus, instrument devant être complété par une série d'autres mesures redistributives.

${ }^{27}$ Guy Standing, Basic Income: And How We Can Make it Happen, Pelican Books, 2017.

28 Yannick Vanderborght, «The Tensions of Welfare State Reform and the Potential of a Universal Basic Income », dans E. Dermine \& D. Dumont (dir.), Activation Policies for the Unemployed, the Right to Work and the Duty to Work, Bruxelles, Peter Lang, 2014, p. 209-222. Au vu de leur travail en commun, il peut sembler étrange de classifier Y. Vanderborght et $\mathrm{P}$. Van Parijs dans des perspectives différentes, mais leurs travaux à quatre mains portent soit sur une introduction générale aux débats, soit sur la version sociale-démocrate.
} 
instrument de renforcement de la protection sociale, à condition que son montant (éventuellement combiné aux autres aides sociales) soit au moins équivalent aux aides sociales existantes. Même un revenu de base modeste, qui préserverait les mêmes montants, le même niveau d'aide sociale, mais offrirait une protection individualisée et inconditionnelle, renforcerait la protection sociale en améliorant le sort des personnes en situation de dépendance (individualisation), en protégeant les chercheurs d'emploi face aux abus de pouvoir ou soupçons d'oisiveté de la part des fonctionnaires publics et en renforçant leur pouvoir de négociation face aux employeurs (inconditionnalité) ${ }^{29}$. À condition que le montant total des prestations sociales ne diminue pas, plus la part du revenu de base dans l'ensemble de ces prestations sera élevée, plus l'État social sera protecteur.

Dans ce modèle, la simplification administrative n'est pas importante en elle-même ${ }^{30}$. L'essentiel est une réorganisation de l'État social qui assure une meilleure protection aux citoyens tout en maintenant une production globale suffisante pour garantir la soutenabilité financière du modèle. Du point de vue social-démocrate, qui est fondamentalement pragmatique, les questions de soutenabilité et de faisabilité sont essentielles. Pour être attrayant, un projet de réforme doit pouvoir être implémenté dans le monde tel qu'il est, avec ses contraintes telles que l'ouverture économique internationale, les difficultés à taxer le capital, ou encore l'exigence de maintenir une certaine compétitivité pour encourager les investissements privés. Cela signifie que les propositions trouvant leur place dans ce modèle de revenu de base «social-démocrate» seront relativement modestes pour des raisons pragmatiques.

Pour cette raison, l'universalité est également optionnelle dans le revenu de base « social-démocrate ». Outre la question du non-recours évoquée plus haut, l'universalité peut séduire dans la mesure où les politiques publiques universalistes ont montré leur plus grande

\footnotetext{
${ }^{29}$ Cet aspect est au cœur des défenses « néo-républicaines » du revenu de base, centrées sur la promotion d'une liberté comme non-domination. Voir par exemple Daniel Raventós, Basic Income: The Material Conditions of Freedom, Londres, Pluto Press, 2007, ainsi que Philip Pettit, « A Republican Right to a Basic Income? », Basic Income Studies, 2/2, 2007.

${ }^{30}$ Daniel Dumont fait valoir de façon convaincante qu'il est illusoire de penser que la simplification de la protection sociale implique nécessairement une amélioration de celle-ci («Le revenu de base universel, avenir de la sécurité sociale ? Une vue sceptique », Revue de Droit Social, 2019/1, p. 151-210). En revanche, il semble légitime de penser qu'un système plus lisible pour les citoyens permettra une protection plus efficace, en réduisant les taux de non-recours.
} 
stabilité dans les États sociaux existants ${ }^{31}$. Plutôt que de cibler une population parfois affublée du stigmate de l'«assistanat », l'universalité élargirait le cercle des personnes mesurant au quotidien les bénéfices d'un système de redistribution continue des revenus du marché. Néanmoins, ce n'est qu'une universalité de façade, puisque dans le scénario le plus probable où le revenu de base est largement financé par l'imposition sur le revenu, ce qui est donné d'une main est repris de l'autre pour les personnes disposant de revenus confortables. Dès lors, dans un esprit pragmatique, on pourrait préférer à cette universalité de façade et aux transferts massifs qu'elle implique une forme de revalorisation des aides sociales et bas revenus, comme dans la formule promulguée entre autres par Thomas Piketty ${ }^{32}$.

\section{Le revenu de transition écologique et sociale}

Le troisième idéal-type peut être associé à une gauche plus radicale, qui se distingue de la gauche sociale-démocrate par des idées et programmes en plus franche rupture avec le statu quo. Dans cet esprit, il ne s'agit pas seulement de préserver l'État social, voire de renforcer quelque peu la protection sociale et atténuer les inégalités ; il s'agit de transformer la société, de la faire évoluer vers une toute autre manière d'organiser la production, voire les échanges.

On peut y associer, outre la figure de Philippe Van Parijs ${ }^{33}$, celles d'Erik Olin Wright ${ }^{34}$ ou André Gorz ${ }^{35}$. Le revenu de base peut séduire, dans une telle perspective, en tant qu'instrument de transition ou de transformation sociale. Hausser les plus bas revenus par le biais d'un impôt progressif ne sera pas nécessairement considéré, dans cette optique, comme le fin mot de la justice sociale, mais cela pourrait être une manière de transformer le capitalisme de l'intérieur. En effet, comme cela a déjà été évoqué, la garantie inconditionnelle de revenu possède potentiellement en elle - à condition que le revenu garanti soit suffisant - un pouvoir de transformation du rapport au travail. Si les citoyens se voient dotés d'un véritable pouvoir

\footnotetext{
${ }^{31}$ Gøsta Esping-Andersen, Les trois mondes de l'État-providence, op. cit. ; Yannick Vanderborght, « The Tensions of Welfare State Reform and the Potential of a Universal Basic Income », art. cité.

${ }^{32}$ Piketty rejette fermement les termes «crédit d'impôt » et «impôt négatif »; il préfère parler de « revenu de base » et de « salaire juste ».

${ }^{33}$ Philippe Van Parijs, Marxism Recycled, Cambridge, Cambridge University Press, p. 155-238 ; Real Freedom for all, op. cit.

${ }^{34}$ Erik Olin Wright, « Basic Income as a Socialist Project », Rutgers Journal of Law \& Urban Policy, 2/1, p. 196203 ; Envisioning Real Utopias, Londres, Verso, 2010, p. 217-222.

${ }^{35}$ André Gorz, Misères du présent. Richesse du possible, Paris, Galilée, 1997, p. 134-161.
} 


\section{Version d'auteur}

de dire non à des emplois qu'ils jugent inadéquats, une des sources fondamentales de l'exploitation - l'obligation matérielle de travailler - disparait ${ }^{36}$. À condition que les emplois les plus précaires ne soient pas déplacés vers les non-citoyens, qui ne jouiraient pas des mêmes droits que les citoyens, cela pourrait radicalement transformer les relations et les conditions de travail en renforçant le pouvoir de négociation des travailleurs ${ }^{37}$.

C'est clairement dans une telle perspective que s'inscrit la fameuse proposition d'une « voie capitaliste vers le communisme » défendue à la fin des années 1980 par Robert van der Veen et Philippe Van Parijs. De leur point de vue, l'introduction d'un revenu de base aussi élevé que possible pourrait amorcer la transition du capitalisme vers le communisme entendu non pas comme la propriété collective des moyens de production, mais comme la satisfaction des besoins de tous et la libération du travail aliéné ${ }^{38}$. Dans un premier temps, le montant du revenu garanti pourrait être relativement modeste afin d'inciter au travail et à la production. Puis, à mesure que se développeraient les forces productives, les incitations à l'effort deviendraient moins importantes et le revenu de base pourrait croître, jusqu'au stade ultime, « communiste », où l'ensemble du produit social serait redistribué en fonction des besoins (via un revenu de base combiné à des aides spécifiques, par exemple) et où le temps pouvant être consacré à des activités non aliénées serait maximisé ${ }^{39}$.

Par ailleurs, le revenu de base pourrait peut-être amorcer la transition vers un horizon plus socialiste en étant utilisé comme un « subside à la grève ${ }^{40}$ » facilitant la mobilisation des travailleurs dans des actions de contestation nécessaires à l'amélioration de leur condition, et en rendant plus aisé le développement d'une économie sociale et solidaire soustraite aux

\footnotetext{
${ }^{36}$ Pierre-Étienne Vandamme, «Exploitation et obligation de travailler », Les Ateliers de l'éthique, 9 (2), p. 29-49 ; Michael Howard, « Exploitation, Labor, and Basic Income », Analyse \& Kritik, 37 (1/2), 2015, p. 281-303. Les travailleurs resteraient néanmoins exploités au sens marxiste originel tant qu'une part du produit de leur travail est accaparée par l'employeur.

${ }^{37}$ Karl Widerquist, Independence, Propertylessness, and Basic Income: A Theory of Freedom as the Power to Say No, New York, Palgrave Macmillan, 2013 ; Yannick Vanderborght \& Philippe Van Parijs, Le revenu de base inconditionnel, op. cit., p. 47-49.

${ }^{38}$ Robert J. van der Veen \& Philippe Van Parijs, « A Capitalist Road to Communism », Theory and Society, 15 (5), 1986, p. 635-655.

${ }^{39}$ Ce scénario repose sur l'idée selon laquelle la question de la propriété (collective ou privée) est secondaire par rapport à l'objectif de satisfaction des besoins et de libération du travail aliéné ; le régime de propriété n'a de valeur qu'instrumentale. (Robert J. van der Veen \& Philippe Van Parijs, « A Capitalist Road to Communism », art. cité, p. 636).

${ }^{40}$ Erik Olin Wright, « Basic Income as a Socialist Project », art. cité, p. 201.
} 
exigences de maximisation du profit ${ }^{41}$. Cela rapproche d'ailleurs ce modèle « socialiste » de celui du revenu de base «écologiste », censé amorcer la transition vers une économie plus soutenable ${ }^{42}$. Les deux se rejoignent largement dans le rapport au travail et l'idée d'une transition vers un tout autre modèle de société. D'ailleurs, tant Van Parijs que Wright et Gorz se soucient à la fois de préoccupations sociales et environnementales.

Dans ce troisième modèle, l'inconditionnalité et la générosité de la garantie de revenu sont donc essentielles, pour accroître le pouvoir des citoyens face à différentes formes de domination, pour leur permettre de travailler moins et pour développer leur pouvoir d'agir. L'universalité, à nouveau, n'est pas absolument essentielle. Elle résonne toutefois bien avec la logique d'une appropriation collective ou socialisation des profits - dans la version socialiste de ce revenu « de transition ». Si la réforme est largement financée par une taxation du capital, comme semble le recommander une inspiration marxiste, l'universalité peut avoir davantage de sens ${ }^{43}$. Toutefois, il ne serait pas non plus incohérent, s'agissant d'un instrument de transition, de cibler les plus bas revenus, c'est-à-dire ceux dont le pouvoir d'agir doit le plus être stimulé.

Les partisans d'un tel revenu de base ne se contenteront cependant pas d'un revenu de base conçu comme unique «correctif» face aux inégalités générées par le capitalisme de marché. S'ils l'adoptent, ce sera soit comme première étape d'un projet plus radical, soit comme complément à d'autres réformes visant la compression des inégalités et une évolution vers des modes de vie soutenables.

\section{Comparer les trois modèles}

\footnotetext{
${ }^{41}$ Chantal Mouffe, The Democratic Paradox, Londres, Verso, 2000, p. 126-127 ; Erik Olin Wright, Envisioning Real Utopias, op. cit., p. 155.

${ }^{42}$ Christian Arnsperger \& Warren E. Johnson, 2011). « The guaranteed income as an equal-opportunity tool in the transition toward sustainability », dans A. Gosseries \& Y. Vanderborght (dir.), Arguing about justice: Essays for Philippe Van Parijs, Louvain-la-Neuve, Presses universitaires de Louvain, p. 61-70; Baptiste Mylondo, Un revenu pour tous! Précis d'utopie réaliste, Paris, Utopia. 2010 ; Sophie Swaton, Pour un revenu de transition écologique, Paris, PUF, 2017.

${ }^{43}$ John Roemer, par exemple, imagine un modèle de socialisme de marché où les profits des firmes seraient redistribués de manière plus ou moins égale à l'ensemble des citoyens sous forme de revenu de base ou « dividende social» («The morality and efficiency of market socialism», dans Egalitarian Perspectives: Essays in Philosophical Economics, Cambridge, Cambridge University Press, 1994, p. 292). Dans ce cas, on voit bien pourquoi l'universalité est importante : pour que les profits soient partagés par l'ensemble de la population.
} 
La distinction entre ces trois idéaux-types permet de mieux mesurer l'absurdité d'un rejet ou d'une acclamation inconditionnels du revenu de base. On ne peut en effet pas porter un jugement normatif identique sur les trois visions politiques déclinées ci-dessus. D’un côté, il s'agit de réduire les dépenses publiques et de flexibiliser le marché de l'emploi, fût-ce au détriment de la protection des citoyens ; de l'autre il s'agit de renforcer cette protection, voire de radicalement transformer les rapports sociaux. Et si l'universalité du revenu n'est plus considérée comme un trait définissant le revenu de base, on ne peut plus affirmer, comme le font beaucoup de critiques de gauche, que seule la version néolibérale est finançable ${ }^{44}$. L'option sociale-démocrate, au moins dans sa version la plus modeste où les niveaux de revenu restent identiques, doit également être finançable sans trop d'efforts ${ }^{45}$ et requiert donc d'être évaluée différemment de la version «néolibérale ».

Ce qui distingue fondamentalement la version «sociale-démocrate» de la version « néolibérale », c'est donc l'idée de renforcer la protection sociale. Les sociaux-démocrates peuvent reconnaître la valeur d'une certaine flexibilité sur le marché de l'emploi, mais qui doit être contrainte par une exigence de sécurité de revenu ${ }^{46}$. Quant à la réduction des dépenses publiques, elle n'est pas un objectif en soi. Ce peut être une contrainte à laquelle font face les gouvernements sociaux-démocrates quand les recettes fiscales sont insuffisantes, mais ce n'est pas une visée de leur modèle. Il s'agit donc de deux modèles bien distincts et incompatibles dans leurs visées. En témoigne notamment le fait que Milton Friedman, défenseur du modèle « néolibéral », refusa en 1968 de signer un appel de 1200 économistes étatsuniens à introduire une forme d'impôt négatif dès lors qu'il trouvait celui-ci trop redistributif et susceptible d'entraîner une réduction «drastique » de l'incitation à travailler ${ }^{47}$. Le revenu de base des uns n'est donc clairement pas celui des autres.

\footnotetext{
${ }^{44}$ Mateo Alaluf, L'allocation universelle, op. cit., p. 80 ; Daniel Zamora, « The Case Against a Basic Income », art. cité.

${ }^{45}$ Je n'entends pas suggérer qu'une allocation véritablement universelle et généreuse soit infinançable. D’un point de vue strictement économique, on peut imaginer une diversité de modèles de financement crédibles (voir Philippe Van Parijs \& Yannick Vanderborght, Le revenu de base inconditionnel, op. cit., p. 221-279). Mais cela impliquerait une augmentation considérable des prélèvements et transferts, qui effraye les gouvernements, un certain nombre de citoyens, et rend politiquement moins plausible l'introduction d'un tel revenu de base dans un avenir proche.

46 Guy Standing, «Meshing Labour Flexibility with Security: An Answer to British Unemployment?», International Labour Review, 125 (1), p. 87-106.

${ }^{47}$ Milton Friedman, There is no such thing as a free lunch, op. cit., p. 198-199.
} 
Quant à la version « de transition écologique et sociale », elle se distingue de la version « sociale-démocrate », nous l'avons vu, par sa plus franche rupture avec le modèle de société existant. Elle se distingue de ce fait par sa moindre faisabilité immédiate. En effet, elle ne peut amorcer de véritable transition vers un autre ordre économique et social qu'à condition de fortement renforcer le pouvoir des plus désavantagés - à condition donc d'offrir une sécurité de revenu très généreuse. À cet égard, ce ne sont pas nécessairement les visées ultimes qui distinguent le modèle « social-démocrate » du modèle « de transition »; ce peut tout aussi bien être le degré de pragmatisme face aux contraintes économiques, sociales et politiques existantes. Autrement dit, on peut adhérer philosophiquement au modèle « de transition » tout en défendant politiquement le modèle «social-démocrate ». C'est d'ailleurs la position de Philippe Van Parijs, qui a développé et défendu, dans des contextes différents, les deux modèles $^{48}$. On pourrait donc considérer qu'entre la version «sociale-démocrate » et la « néolibérale », il y a une différence de nature, tandis qu'entre la « sociale-démocrate » et celle « de transition», il y aurait une différence de degré (de radicalisme ou de pragmatisme). Soulignons néanmoins que certains défenseurs du modèle social-démocrate ne veulent peutêtre pas d'une rupture franche avec le statu quo et seraient pleinement heureux avec un État social protecteur garantissant la paix sociale et limitant les inégalités de marché, sans condamnation plus globale du capitalisme ou des échanges marchands. Le modèle «socialdémocrate » peut donc être défendu de deux points de vue. Soit, comme un idéal en soi, soit comme une première étape, en attendant des conditions plus favorables à une véritable transition écologique et sociale, voire une sortie du capitalisme.

\section{Conclusion : quelle radicalité ?}

Le modèle «néolibéral » est radical dans la simplification de l'État providence qu'il propose. L'idée est d'en finir avec la bureaucratie entourant les politiques sociales, de réduire le nombre de fonctionnaires nécessaires à l'administration de celles-ci, et d'ainsi limiter les fonctions de l'État à quelques interventions ciblées permettant la fluidité du marché de l'emploi et une cohésion sociale minimale favorable au dynamisme économique.

\footnotetext{
${ }^{48}$ Pour ce qui concerne le modèle «social-démocrate», voir Philippe Van Parijs, «Basic Income and Social Democracy », Social Europe, 11, 2016 ; Philippe Van Parijs \& Yannick Vanderborght, « From Euro-stipendium to Euro-dividend », Journal of European Social Policy, 11 (4), p. 342-346.
} 
À l'inverse, le modèle « de transition » est radical dans ses visées de transformation des relations sociales et des dynamiques économiques. Il vise en effet une plus grande égalité entre les citoyens, une libération par rapport au travail aliéné, ainsi qu'une diminution volontaire de la production quand la visée écologiste s'ajoute à la visée sociale. Il s'agit donc de deux formes de radicalité qui n'ont rien en commun. Le rapport à l'État est différent. Le rapport au capitalisme et au marché est différent. Et les visées ultimes sont incomparables.

Le modèle «social-démocrate », quant à lui, n'entend pas révolutionner la protection sociale, introduire une modification radicale, mais proposer une réforme qui, tout en étant plus réaliste à court-terme, est susceptible d'initier une transformation plus graduelle de l'État social et des relations marchandes ${ }^{49}$. Ses promoteurs gagneraient cependant à clarifier le statut de leur proposition, notamment pour contrer les critiques de gauche qui pointent précisément du doigt son manque de radicalité ou sa relative insensibilité aux inégalités de marché. Un tel revenu de base suffirait-il à rendre nos sociétés justes ? Qu'est-ce qui justifie les inégalités restantes ? S'agit-il au contraire d'un premier pas pragmatique vers une transformation plus radicale? D'un aspect d'un projet de réforme plus large et plus ambitieux (comme celui esquissé par Thomas Piketty dans Capital et idéologie, par exemple) ?

Ce qui est toutefois radical dans l'ensemble des trois modèles, c'est l'inconditionnalité, qui rompt complètement avec la logique tant de l'assurance sociale que celle implicite de l'assistance sociale. En effet, contrairement à une idée reçue, les modèles existants n'ont jamais été conçus comme offrant une aide inconditionnelle aux citoyens ne travaillant pas ${ }^{50}$. L'obligation de travailler ou au moins de chercher du travail a toujours été l'arrière-plan des politiques sociales, dans tous les États sociaux. Or, tous les modèles de revenu de base rompent radicalement avec cette logique.

Néanmoins, dans le modèle «néolibéral», comme nous l'avons vu, cette inconditionnalité est fragile. Elle n'est généralement pas justifiée par un droit inconditionnel à

\footnotetext{
${ }^{49}$ On pourrait imaginer dans le même esprit un modèle de droite modérée, qui sans vouloir faire table rase de l'État social, chercherait à l'amincir graduellement, par la fusion des diverses prestations sociales. Un bon exemple est sans doute l'impôt négatif préconisé par Koenig et de Basquiat (LIBER, un revenu de liberté pour tous, op. cit.). Je laisse toutefois ce modèle de côté, dans cet article, parce qu'il est beaucoup moins présent dans les débats académiques et politiques.

${ }^{50}$ Voir Daniel Dumont, «Activation rime-t-elle nécessairement avec stigmatisation ? Une mise en perspective critique du procès de l'État social actif », Droit et société, 78 (2), 2011, p. 449-456.
} 
la sécurité de revenu ${ }^{51}$; plutôt par l'hypothèse empirique qu'il est plus simple et moins coûteux de garantir le revenu que d'essayer de distinguer les pauvres «méritants » des «paresseux ». La radicalité de la rupture de ce modèle avec la logique dominante est par ailleurs atténuée par le faible montant du revenu : on sait que les personnes devront de toute façon travailler pour vivre. La rupture avec l'attente de réciprocité n'est donc pas franche.

Mais il y a donc bien un aspect radical même dans le modèle a priori le plus modeste : le modèle «social-démocrate ». Si l'idée d'inconditionnalité devait un jour s'imposer dans un État social, si l'on décidait de donner la priorité absolue à la satisfaction des besoins de tous par rapport à l'attente de réciprocité ${ }^{52}$, il s'agirait déjà d'une rupture radicale dans l'histoire de la protection sociale. La raison est dévoilée sans complexe dans cette affirmation d'un marchand écossais au début du $19^{\mathrm{e}}$ siècle : «Sans une proportion importante de pauvreté, il n’y aurait pas de richesses, puisque les richesses sont le fruit du travail, tandis que le travail ne peut résulter que d'un état de pauvreté 53 » 54 .

Résumé - Parler du revenu de base (revenu universel, allocation universelle) au singulier n'a pas beaucoup de sens. Évaluer une telle proposition dans l'abstraction, sans préciser de quel modèle de revenu de base on parle, encore moins. Il s'agit en effet d'un instrument pouvant être mis au service d'une multitude de causes et aux effets très variés selon le projet politique présidant à son implémentation. Cet article propose pour cette raison de distinguer trois idéaux-

\footnotetext{
51 À l'exception de Matt Zwolinski, qui outre un argument «pragmatique » pour le revenu de base («The pragmatic libertarian case for a basic income guarantee », Cato Unbound, 4 août 2014), très proche de celui de Friedman, défend également un droit égal à la valeur brute des ressources naturelles, dans une argumentation plus proche du courant libertarien de gauche («A moral case for Universal Basic Income », art. cité).

${ }^{52}$ Pour des critiques de la priorisation de la réciprocité dans des sociétés très inégales, voir notamment Karl Widerquist, « Reciprocity and the Guaranteed Income », Politics \& Society, 27 (3), 1999, p. 387-402 ; PierreÉtienne Vandamme, «Democracy and the Right to Income », Astrolabio: revista internacional de filosofia, 19, 2017, p. 162-183 ; Marc-Antoine Sabaté, « Le revenu de base : renversement ou renouveau du droit social ?», op. cit., ch. 9-10 ; Philippe Van Parijs \& Yannick Vanderborght, Le revenu de base inconditionnel, op. cit., p. 172174.

53 Patrick Colquhoun, cité dans Thomas H. Marshall, Class, Citizenship and Social Development, Westport, Greenwood, 1973, p. 86.

${ }^{54}$ Cet article doit beaucoup aux discussions que j'ai pu avoir avec Daniel Dumont, Marc-Antoine Sabaté, Nabil Sheikh Hassan, Yannick Vanderborght, Philippe Van Parijs et Daniel Zamora. Je remercie également Victor Mardellat et les personnes qui ont évalué cet article pour leurs précieuses suggestions.
} 
types de revenu de base : néolibéral, social-démocrate et de transition écologique et sociale. Ces idéaux-types se distinguent par leurs caractéristiques internes (montant ; financement ; articulation avec les aides sociales existantes) et par les projets politiques qui les guident. La question de la radicalité du revenu de base dépend donc du modèle envisagé. Le modèle « néolibéral » est radical dans la simplification de l'État providence qu'il propose, tandis que le modèle « de transition » l'est dans ses visées de transformation des relations sociales et des dynamiques économiques. Le modèle «social-démocrate», quant à lui, n'entend pas révolutionner la protection sociale, mais constituer une réforme qui, tout en étant plus réaliste à court-terme, est susceptible d'initier une transformation plus graduelle des États sociaux dans le sens d'un renforcement de la protection sociale.

\begin{abstract}
Talking about basic income in the singular does not make much sense. Evaluating such a proposition in the abstract, without specifying what kind of basic income we are talking about, even less so. For it is an instrument that can be used for a diversity of purposes and with varied effects depending on the political project guiding its implementation. Therefore, this article proposes to distinguish between three ideal types of basic income: neoliberal, socialdemocratic, and of ecological and social transition. These ideal types distinguish themselves by their internal characteristics (amount; financing; articulation with existing social protection) and by the political projects that guide them. The question of the radicality of the basic income proposal thus depends on the model envisaged. The "neoliberal" model is radical in the simplification of the welfare state it proposes, while the "transition" model is radical in its aim to transform social relations and economic dynamics. The "social-democratic" model, in contrast, does not intend to revolutionize social protection, but rather to introduce a reform that, while being more realistic in the short term, is likely to initiate a more gradual transformation of welfare states towards a strengthening of social protection.
\end{abstract}

